\title{
Thermal and Non-Thermal Emission in Gamma-ray Bursts: GRB 090902B as a Case Study
}

\author{
Sinéad McGlynn* \\ Oskar Klein Centre/Dept. of Physics, Royal Institute of Technology (KTH), Stockholm, Sweden \\ E-mail: smcglynn@particle.kth.se
}

\section{Felix Ryde}

Oskar Klein Centre/Dept. of Physics, Royal Institute of Technology (KTH), Stockholm, Sweden E-mail: felix@particle.kth.se

\section{Christoffer Lundman}

Oskar Klein Centre/Dept. of Physics, Royal Institute of Technology (KTH), Stockholm, Sweden

E-mail: clundman@particle.kth.se

\section{Asaf Pe'er}

Space Telescope Science Institute, Baltimore, MD, USA/Harvard-Smithsonian Center for

Astrophysics, Cambridge, MA, USA

E-mail: apeer@cfa.harvard.edu

\begin{abstract}
Photospheric (thermal) emission is inherent to the gamma-ray burst (GRB) "fireball" model. Inclusion of this component in the analysis of the GRB prompt emission explains some of the prompt GRB spectra seen by the Fermi satellite over its entire energy band. The sub-MeV peak corresponds to multi-color black body emission, and the high energy tail results from similar contributions of synchrotron emission, synchrotron self Compton (SSC) and Comptonization of the thermal photons by energetic electrons originating after dissipation of the kinetic energy above the photosphere. We study the connection between the thermal and non-thermal parts of the spectrum, and deduce the values of the free model parameters from the data. We demonstrate our analysis method on GRB 090902B.
\end{abstract}

8th INTEGRAL Workshop "The Restless Gamma-ray Universe"

September 27-30, 2010

Dublin, Ireland

\footnotetext{
${ }^{*}$ Speaker.
} 


\section{Introduction}

The bright, long GRB 090902B ( $z=1.822)$ was detected by both instruments on board Fermi and was one of the brightest observed to date by the Large Area Telescope (LAT; [1]). The prompt spectrum of GRB 090902B over the energy range $8 \mathrm{keV}-10 \mathrm{GeV}$ shows a clear deviation from the generally expected Band function [2].

It has been suggested [10] that GRB spectra are a superposition of two spectral components: photospheric blackbody emission and an accompanying non-thermal component. We investigated the peaked component of the spectrum, i.e. the Band component, at a higher time resolution than in previous works [1]. These time bins were chosen such that a reasonable signal to noise ratio was obtained for each GBM detector.

\section{Fitting}

The blackbody temperature is generally expected to vary with latitude angle. The optical depth is also angle dependent, resulting in the photospheric radius increasing with angle. Angle dependent density profiles of the outflow will have a similar effect. It is therefore plausible that the photospheric component is better represented by a multicolor blackbody instead of a single Planck function. We therefore fit the photospheric component with a multicolor blackbody (mBB), which is given by:

$$
F^{m B B}\left(E, T_{\max }\right) \propto \int_{T_{\min }}^{T_{\max }} \frac{d A(T)}{d T} \frac{E^{3}}{\exp [E / k T]-1} d T
$$

The spectral shape consists of a superposition of Planck functions in the temperature range $T_{\min }-T_{\max }$ where $T_{\max }$ is a free parameter and $T_{\min }$ is not determined in the fit. The total flux is determined to be

$$
F(T)=F_{\max }\left(\frac{T}{T_{\max }}\right)^{q} \propto A(T) T^{4}
$$

The fit for one time interval $(t=11.008-11.392 \mathrm{~s})$ is shown in Figure 1. The inclusion of the thermal emission in the prompt emission spectra produces a complete, self consistent physical model of the prompt emission spectrum seen over the entire Fermi energy range [7]. This is in contrast to the Band function fits, which do not provide any physical interpretation and require extra models to be able to fit the spectrum at high energies. The theory behind multicolor blackbody emission is explained in more detail elsewhere [8].

When observing the photosphere we see a superposition of spectra with different temperatures as measured in the observer frame. Several effects will cause the low energy slope to differ from that of a simple black body:

1. The observed temperature is a function of angle with respect to the line-of-sight. Integrating the emitting surface over angle will produce a multicolor black body spectrum. At late times, high latitude effects produce a spectrum with $\alpha=-1$ [11]. 


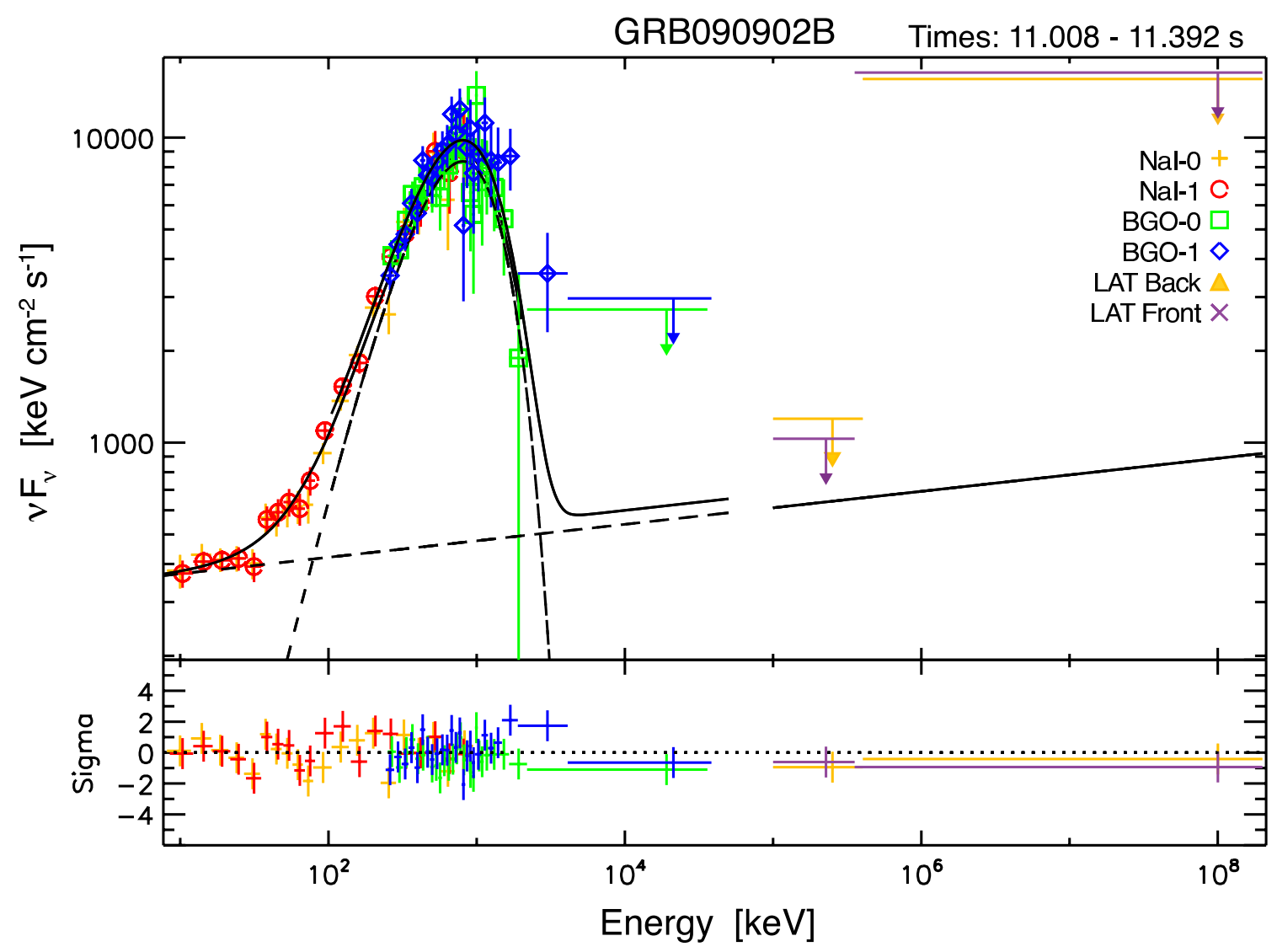

Figure 1: Time-resolved $v F_{v}$ spectrum for the interval $t=11.008-11.392 \mathrm{~s}$ over the GBM+LAT energy ranges, fit with a mBB+power-law model. Different datasets are represented as in the legend (top right).

2. Depending on how the comoving outflow density scales with radius, the probability density function for a photon to make its last scattering at radius $r$ before reaching the observer does not have to be extremely sharply peaked around $r(\tau=1)$ [6]. If the comoving outflow temperature or Lorentz factor varies on distance scales of the width of the probability density profile peak, integrating over the radius is necessary to get the observed spectrum.

3. The outflow properties around the photosphere are expected to vary on a time-scale shorter than the most highly time-resolved spectra [9]. Thus, the observed spectra are most likely to be the result of an integration over time, which can produce a soft low energy slope for reasonable scalings of the emitting surface and temperature with time [3].

These effects have the potential of softening the low energy slope from purely thermal emission as well as producing a somewhat wider peak in the spectrum. Simulations are needed in order to determine the relative importance of these effects. Indeed recent simulations from [4] find thermal emission from a GRB jet with $\alpha=-0.5$, much softer than a black body spectrum with $\alpha=1$. 


\section{Photospheric Properties}

According to the model presented here, the sub-MeV peak is interpreted as multi-color black body emission from the photosphere. The non-thermal, high energy part seen in several bursts by the LAT is interpreted as combined emission of synchrotron, SSC and Comptonization of the thermal photons, following an episode of energy dissipation that occurs at a large radius above the photosphere. Observations of high energy photons can be used to estimate the dissipation radius $r_{\gamma}$ :

$$
r_{\gamma}=3 \times 10^{15} L_{54}^{1 / 2} \delta t_{0}^{o b 1 / 2} \varepsilon_{\max , 10}^{o b}{ }^{1 / 2} \eta_{3}^{-1} \mathrm{~cm}
$$

which is generally above the photospheric radius $r_{p h}$, where the flow becomes optically thin [12]:

$$
r_{p h}=\left[\frac{d_{L}^{5} F_{T h}^{o b} \sigma_{T} R^{3}}{\xi^{3}(1+z)^{6} 2 m_{p} c^{3}}\right]^{1 / 4}\left(\frac{L}{L_{T h}^{o b}}\right)^{1 / 4}
$$

Here, the dimensionless parameter $R$ is related to the effective size of the photosphere:

$$
R=\left(\frac{F_{T h}^{o b}}{\sigma T^{o b 4}}\right)^{1 / 2}=\xi \frac{(1+z)^{2}}{d_{L}} \frac{r_{p h}}{\eta}
$$

The Lorentz factor $\eta$ can then be determined using the equation:

$$
\eta=\left[\xi(1+z)^{2} d_{L} \frac{F_{T h}^{o b} \sigma_{T}}{2 m_{p} c^{3} R}\right]^{1 / 4}\left(\frac{L}{L_{T h}^{o b}}\right)^{1 / 4}
$$

\section{Discussion}

The separation between thermal and non-thermal emission makes it possible to deduce the values of the free model parameters. By analysing the photospheric part of the spectrum, the Lorentz factor and photospheric radius can be determined. The Lorentz factor was determined to be in the range $780 \leq \eta \leq 1000$, with photospheric radius $r_{p h} \sim 6.1-7.8 \times 10^{11} \mathrm{~cm}$.

By analysing the non-thermal part of the spectrum, one can constrain the dissipation radius to be larger than $4.0-5.0 \times 10^{15} \mathrm{~cm}$. For parameters characterising LAT GRBs, the emission radius of the non-thermal photons is 3-4 orders of magnitude higher than the photospheric radius, indicating that the observed spectrum must be emitted from at least two regions of emission. This is consistent with the lag of $0.5 \mathrm{~s}$ between the light curve of the thermal emission and the light curve of the nonthermal emission [11].

Only the non-thermal part of the spectrum is required to originate from energy dissipation above the photosphere. This dissipation process is expected to produce a power-law distribution of energetic electrons with index $p \geq 2.0$. Comparison of the cooling time to the dynamical time scale implies that the electron population is in the fast cooling regime. This fast cooling implies that virtually all of the dissipated energy given to the electrons is radiated.

The relative contributions of synchrotron emission, SSC and Comptonization of the thermal photos are expected to be of the same order of magnitude. Hence, the three emission mechanisms 
have roughly similar contributions to the high energy part of the spectrum. This makes it difficult to directly determine the power-law index of the accelerated electrons from measurements of the high energy spectral slope.

GRB 090902B has a clear separation between the thermal and non-thermal components and a pronounced thermal peak: both above and below the peak, the spectral slopes are too steep to enable fitting the spectrum with any combination of synchrotron and SSC emission models. However, excellent fits are obtained using the thermal + non-thermal model. By doing so, we were able to constrain the hydrodynamic properties of the outflow and the physical properties at the emission site. The high efficiency required may hint towards a Poynting dominated outflow, i.e. the most plausible source of energy may be magnetic [12].

\section{Conclusions}

GRB 090902B shows a very pronounced peak and clearly separated high energy component. Generally, the observed sub-MeV peak is not as pronounced as in GRB 090902B: the low and high energy spectral slopes are not as steep, and a pure single black body spectrum is too narrow to fit the peak. However, there are two effects which are often neglected. First, the fits are made to time integrated spectra. As the properties of the outflow, in particular the Lorentz factor, vary on a very short timescale, the black body spectrum is often smeared. Also, at any given instance, an observer sees thermal photons emitted simultaneously from different radii and different angles to the line of sight. As a result, the expected photospheric emission is not a pure black body, but a combination of black body spectra with different amplitudes. Thus one expects to see a multi-color black body, as observed here. An alternative explanation may be inverse Compton scattering by electrons which are accelerated close to the photosphere, which can also produce several spectra [5].

\section{References}

[1] Abdo, A.A. et al. (2009). ApJL, 706, L138.

[2] Band, D. et al. (1993). ApJ, 413, 281.

[3] Blinnikov S. I., Kozyreva A. V. \& Panchenko I. E. (1999). ARep, 43, 739.

[4] Mizuta A., Nagataki S. \& Aoi J. (2010). arXiv astro-ph:1006.2440.

[5] Pe’er, A., Mészáros, P. \& Rees, M. J. (2006). ApJ, 642, 995.

[6] Pe'er A. (2008). ApJ, 682, 463.

[7] Pe'er, A. et al. (2010). arXiv astro-ph:1007.2228.

[8] Pe'er, A. \& Ryde, F. (2010). arXiv astro-ph:1008.4590.

[9] Rees, M. J. \& Mészáros P. (2005). ApJ, 628, 847.

[10] Ryde, F. (2005). ApJ, 625, L95.

[11] Ryde, F. et al. (2010). ApJ, 709, L172.

[12] Zhang, B. \& Pe'er, A. (2009). ApJL, 700, L65. 